\title{
Anthraquinone Derivatives as Potent Inhibitors of c-Met Kinase and the Extracellular Signaling Pathway
}

Zhongjie Liang, ${ }^{\dagger \neq}$ Jing Ai, ${ }^{\ddagger}$ Xiao Ding, ${ }^{\ddagger}$ Xia Peng, ${ }^{\ddagger}$ Dengyou Zhang, ${ }^{\ddagger}$ Ruihan Zhang, ${ }^{\ddagger}$ Ying Wang,

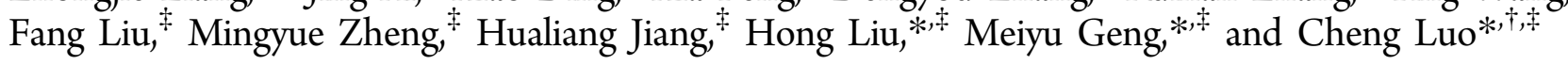

${ }^{\dagger}$ Center for Systems Biology, Soochow University, Suzhou 215006, China

${ }^{\ddagger}$ State Key Laboratory of Drug Research, Shanghai Institute of Materia Medica, Chinese Academy of Sciences, Shanghai 201203, China

Supporting Information

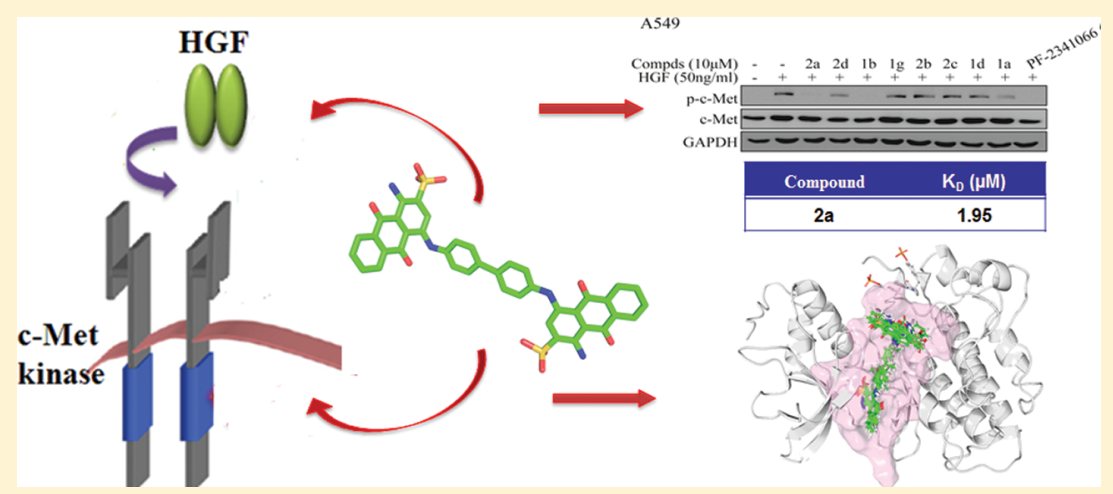

ABSTRACT: The aberrant function of c-Met kinase signaling pathway is ubiquitously involved in a broad spectrum of human cancers; thus, a strong rationale exists for targeting the kinase pathway in cancer therapy. Via integration of computational and experimental studies, anthraquinone derivatives were identified for the first time as potent c-Met kinase inhibitors in this research. The aberrant activation of the c-Met kinase pathway results from (TPR)-Met, MET gene mutation, or amplification and a hepatocyte growth factor (HGF)/scatter factor-dependent autocrine or paracrine mechanism. However, anthraquinone derivatives exclusively suppressed c-Met phosphorylation stimulated by HGF in A549 cells, indicating that the compounds possess the ability to block the extracellular HGF-dependent pathway. A surface plasmon resonance assay revealed that the most potent compound, 2a, shows a high binding affinity for HGF with an equilibrium dissociation constant of $1.95 \mu \mathrm{M}$. The dual roles of compound $\mathbf{2 a}$ demonstrate the potency of anthraquinone derivatives and provide a new design solution for the c-Met kinase signaling pathway.

KEYWORDS: Anthraquinone derivatives, c-Met kinase inhibitors, binding affinity with HGF

$\mathrm{T}$ he receptor tyrosine kinase c-Met is the product of the MET proto-oncogene. c-Met kinase, together with its natural ligand, the growth and motility factor hepatocyte growth factor/scatter factor (HGF/SF), provides essential signals for cell survival and the long distance migration of epithelial and myogenic precursor cells during embryonic development and tissue morphogenesis. HGF/SF-induced cMet dimerization activates the receptor tyrosine kinase by phosphorylation of tyrosine residues (e.g., Tyr1230, Tyr1234, and Tyr1235) in the kinase domain. Consequent phosphorylation and recruitment activate various downstream signaling cascades, including the RAS-MAPK and PI3K-AKT branches, both of which critically affect cell scattering and invasion and protect cells from apoptosis and angiogenesis. ${ }^{1}$ The aberrant activation of c-Met kinase signaling in human cancers can be induced by specific genetic lesions, transcriptional upregulation, or HGF/SF-dependent autocrine or paracrine mechanisms. Specifically, c-Met protein overexpression resulting from amplification of the $M E T$ gene or transcriptional upregulation is the most frequent mechanism of constitutive kinase activation. The MET gene can also carry activating mutations independent of ligand activation. Translocated promoter region (TRP)-Met, which is derived from chromosomal rearrangement, can be constitutively activated because of structural dimerization provided by the TPR moiety. Besides, the natural ligand of c-Met, HGF, can activate c-Met kinase aberrantly in an autocrine fashion, yielding the HGF-dependent activation. Considering the various consequent signaling cascades involved, HGF/SF-c-Met signaling is frequently hijacked by cancer cells for survival and metastasis, thus providing these molecules as versatile targets for cancer therapy. ${ }^{2,3}$

Received: January 4, 2013

Accepted: February 25, 2013

Published: February 25, 2013 
Given our improved understanding of structure-function relationships in the c-Met kinase pathway, considerable progress in the development of inhibitors for cancer therapy has been made. ${ }^{4-8}$ To date, several strategies have been pursued to identify inhibitors of the kinase signaling pathway, each of which focuses on one of the serial steps regulating cMet activation. Biological antagonists and antibodies are involved in blocking the extracellular interactions between cMet and the HGF ligand. Small-molecule compounds are believed to occupy the active site of the c-Met kinase domain, thereby prohibiting downstream phosphorylation. ${ }^{9}$ This strategy has captured extensive attention as a consequence of potential inhibitor compounds reaching various clinical testing stages. ${ }^{10}$ The success of Pfizer's PF-2341066 (crizotinib) in clinical treatments confirms the rationality of this strategy. ${ }^{11}$

Today, the universal success in the discovery of smallmolecule inhibitors against kinase has yielded a wealth of structural data but has led to a highly congested intellectual property space. Pharmacophore-based virtual screening is an effective approach for identifying new chemical entities from known active compounds. ${ }^{12-14}$ The computational pharmacophore approach has dramatically accelerated the speed of drug discovery, making it a favorable method for our study. ${ }^{15} \mathrm{We}$ employed the rational pharmacophore model to discover new chemical entities from the SPECS database. Through hybrid computational and experimental methods, the details of which have been previously published, ${ }^{16}$ potent candidates against cMet kinase were identified. Compound 1a was identified as a novel effective inhibitor [inhibitory concentration $\left(\mathrm{IC}_{50}\right)$ of 9.5 $\mu \mathrm{M}$ ] (Figure 1). Then, via substructure similarity research



Figure 1. Chemical structure of compound 1a.

against the SPECS database and chemical synthesis, a series of derivatives were evaluated as c-Met kinase inhibitors, the results of which demonstrate that anthraquinone is an effective inhibitory scaffold for the kinase (Tables 1 and 2).

The synthesis of these compounds is depicted in Scheme 1. Treatment of sodium 1-amino-4-bromo-9,10-dioxo-9,10-dihydroanthracene-2-sulfonate with 1 equiv of diversely substituted amines in phosphate buffer $(\mathrm{pH} \mathrm{6-7)}$ in the presence of a catalytic amount of copper powder $\left(\mathrm{Cu}^{0}\right)$ under microwave irradiation at $80^{\circ} \mathrm{C}$ for $20 \mathrm{~min}$ afforded target compounds $1 \mathrm{a}-$ $\mathbf{m}$ in moderate to excellent yields (Scheme 1). Compounds $\mathbf{2 a}-\mathbf{h}$ were obtained from the microwave-assisted Ullmann coupling of sodium 1-amino-4-bromo-9,10-dioxo-9,10-dihydroanthracene-2-sulfonate with 0.5 equiv of diversely substituted amines at $120{ }^{\circ} \mathrm{C}$ for $20 \mathrm{~min}$. The compounds were purified by flash reversed column chromatography, and their purity (at least 90\%) was determined by high-performance liquid chromatography-mass spectrometry/ultraviolet methods. Details of the synthetic procedures and structural characterizations are described in the Supporting Information.
Table 1. Chemical Structures of Target Compounds $1 \mathrm{a}-\mathrm{m}$ and Their c-Met Kinase Inhibitory Activities

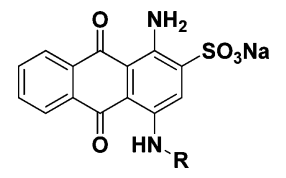

Inhibition (\%)

The primary inhibitory activities of the synthesized compounds at $10 \mu \mathrm{M}$ against c-Met kinase were determined. According to preliminary results, eight compounds of the synthesized derivatives (i.e., $\mathbf{1 a}, \mathbf{1} \mathbf{b}, \mathbf{1 d}, \mathbf{1 g}$, and $\mathbf{2 a - d}$ ) showed favorable inhibitory activities $(>50 \%)$, and their $\mathrm{IC}_{50}$ values were further measured. Tables 1 and 2 show the results of these determinations. The detailed bioassay protocol is described in Experimental Procedures. Assay results indicated that compounds with benzidine (1a) and $o$-toluidine (1) substituted at the C-4 position show moderate inhibitory activities against cMet kinase. By contrast, acetyl (1d) and pyrimidin-2-yl (1g) groups showed an increase in inhibitory activity compared with the value with a benzyl group at the same position. Introducing the benzyl (1c), tert-butoxycarbonyl (1e), and cyclohexanecarbonyl (1f) groups here decreased inhibitory activities with enzyme potencies of $25.1,28.0$, and $29.2 \%$, respectively. Increasing the length of the group by replacing the benzidine 
Table 2. Chemical Structures of Target Compounds $2 a-h$ and Their c-Met Kinase Inhibitory Activities

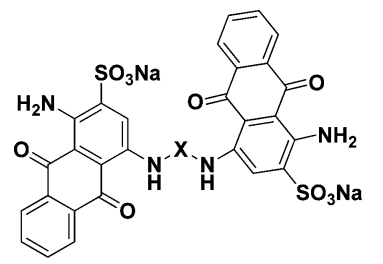

Compound Inhibition (\%)

group with 4,4'-methylenedianiline (1h), 4,4' -oxidianiline (1i), and $4,4^{\prime}$-(ethane-1,2-diyl)dianiline (1j) groups also decreased inhibitory activity. Changing the benzidine group to 2,5dimethylbenzene-1,4-diamine (1k), 2,5-dimethylbenzene-1,4diamine (11), and aniline (1m) groups weakened the inhibitory activity of the molecules to $21.1,40.8$, and $32.7 \%$, respectively.

For compounds $\mathbf{2 a}-\mathbf{h}$, vicarious links between two anthraquinone rings were investigated. Among these derivatives, the biphenyl group proved to be the most favorable substituent for link X (2a-d). Compounds with biphenyl (2a), 2,2'-dimethylbiphenyl (2b), 2,2'-dimethoxybiphenyl (2c), and $3,3^{\prime}$-dimethylbiphenyl (2d) groups showed potent inhibitory activities of $82.9,91.4,97.0$, and $76.5 \%$, respectively. Replacement of the biphenyl group with longer groups, such as methylenebis(4,1-phenyl) (2e) or oxybis(4,1-phenyl) (2f), dramatically decreased inhibitory activities. Likewise, replacement of the biphenyl group with shorter groups, such as phenyl $(\mathbf{2 g})$ or 2,5-dimethyl-1,4-phenyl (2h), also eliminated inhibitory activity.

Through the structure-activity relationship (SAR) analysis, we further examined the binding interactions of anthraquinone derivatives with c-Met kinase. For docking simulations, the autophosphorylated c-Met kinase structure (Protein Data Bank entry $3 \mathrm{R} 7 \mathrm{O})^{17}$ was selected as the docking model for our anthraquinone compounds. As this autophosphorylated structure leads to the ejection of the activation loop and rearrangement of helix aC, which contributes to an enlarged active site, the enlarged pocket is quite compatible of our anthraquinone derivatives here. The result of the simulation showed that anthraquinone derivatives not only resided in the adenosine triphosphate (ATP) site but also extended toward the activation loop, which suggests their selectivity toward autophosphorylated c-Met kinase (Figure 2A). Overall, intramolecular $\mathrm{H}$-bonds between the carbonyl and amino groups maintained the consistent conformation of anthraquinone derivatives, facilitating their binding poses in the pocket. As expected, the 1-amino and 9-carbonyl groups H-bonded with Pro1158 and Met1160 residues in the hinge region. The A-ring of anthraquinone partially formed $\pi-\pi$ stacking interactions with the Tyr1159 side chain.

The anthraquinone rings were also involved in favorable hydrophobic interactions with the surrounding residues Ile1084, Val1092, Ala1108, Leu1140, Leu1157, Met1211, and Ala1221. The E-ring extended toward the activation loop, with the terminal amino of compound 1a H-bonding with His 1088 and Asp1204 (Figure 2B). Polar residues Arg1086, Asp1204, Asn 1209, and Asp1222 around the E -ring indicate that the introduction of polar substituents into the molecule increases potency. Conversely, the introduction of hydrophobic substituents into the molecule decreases activity, consistent with the previously demonstrated poor activity of compounds 1c, 1e, and 1f. The second anthraquinone ring of compound 2a resided toward the phosphorylated flexible loop, pointing the sulfonic acid group into the solvent-exposed area in an enthalpy-driven process (Figure 2C). Because the length of anthraquinone derivatives with the biphenyl group as the link $\mathrm{X}$ is perfectly suited to the pocket, other fragments as links may not be beneficial for activity, consistent with the SAR analysis.

Subsequently, the in vitro c-Met targeting activity of anthraquinone derivatives was investigated. Cell lines with different mechanisms of c-Met activation were selected.

Scheme 1. Synthesis of Target Compounds $1 \mathrm{a}-\mathrm{m}$ and $2 \mathrm{a}-\mathrm{h}^{a}$



${ }^{a}$ Reaction conditions: (a) $\mathrm{RNH}_{2}$ (2 equiv), $\mathrm{Cu}, \mathrm{NaH}_{2} \mathrm{PO}_{4} / \mathrm{Na}_{2} \mathrm{HPO}_{4}, \mathrm{H}_{2} \mathrm{O}, \mathrm{MW}, 80{ }^{\circ} \mathrm{C}$; (b) $\mathrm{NH}_{2}-\mathrm{X}-\mathrm{NH}_{2}\left(0.5\right.$ equiv), $\mathrm{Cu}, \mathrm{NaH}_{2} \mathrm{PO} 4 / \mathrm{Na}_{2} \mathrm{HPO}_{4}$, $\mathrm{H}_{2} \mathrm{O}, \mathrm{MW}, 120^{\circ} \mathrm{C}$. 

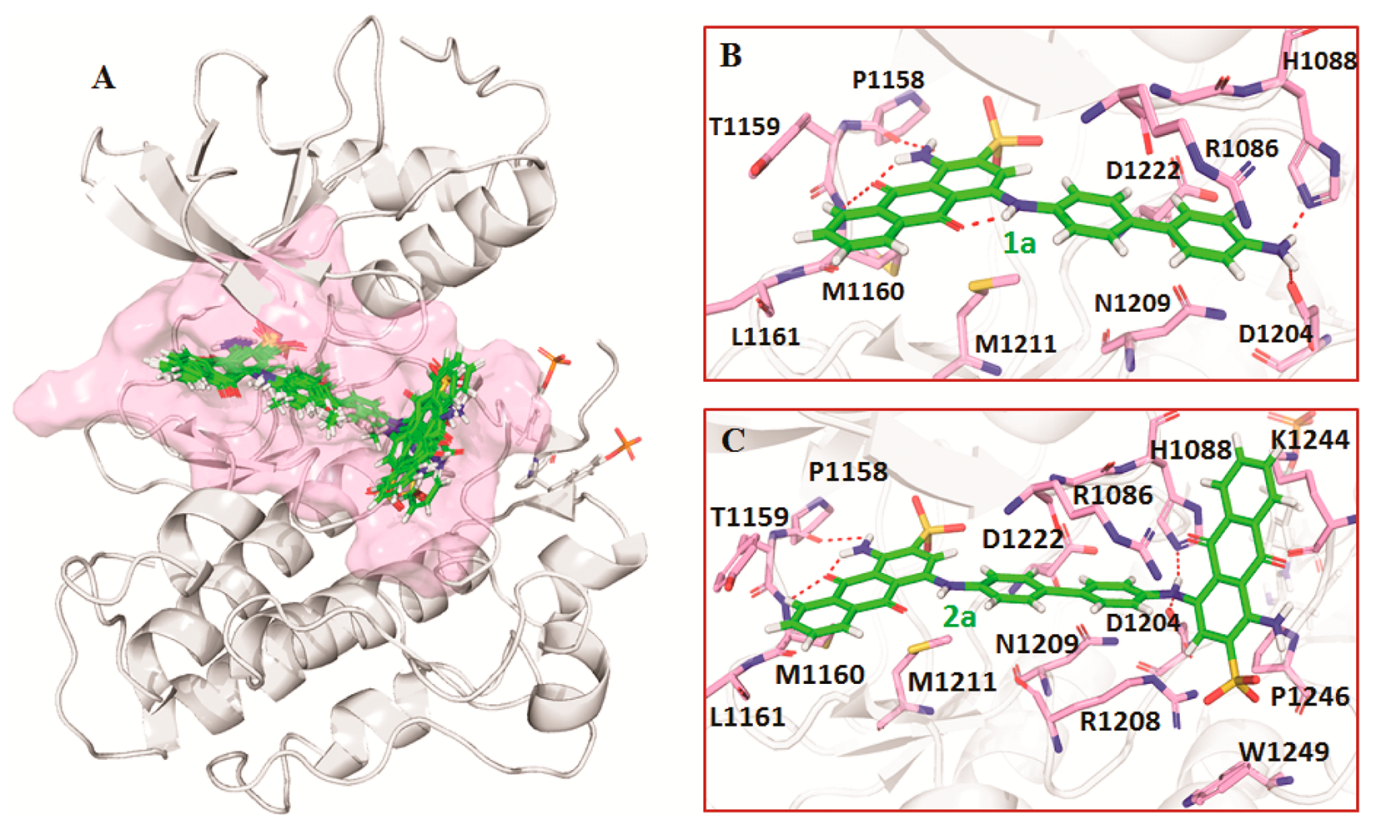

Figure 2. (A) Docking simulations show that anthraquinone derivatives occupy the ATP site of c-Met kinase. Anthraquinone derivatives are shown as green sticks and surrounding residues are colored pink. (B) Detailed mode of binding of compound 1a with c-Met kinase. (C) Detailed mode of binding of compound 2a with c-Met kinase.

Compounds 2a, 2d, 1b, and 1a markedly inhibited HGFinduced c-Met phosphorylation at a dosage of $10 \mu \mathrm{M}$ in A549 cells, which naturally express c-Met and respond to HGF stimulation (Figure 3A). As the activation of c-Met might be HGF-dependent or HGF-independent manner, we extended our study to BaF3/TPR-Met and MKN45 cell lines, in which cMet activation is HGF independent. BaF3/TPR-Met cells stably express a constitutively active and oncogenic form of cMet derived from chromosomal rearrangement, whereas
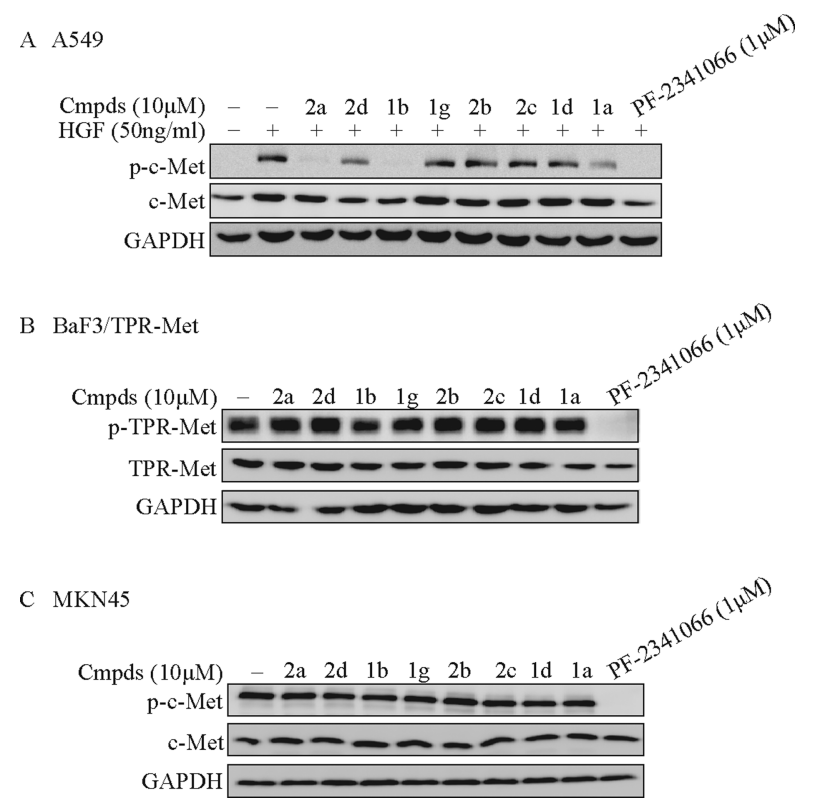

Figure 3. Anthraquinone derivatives inhibited HGF-induced c-Met phosphorylation. (A) Anthraquinone derivatives suppressed c-Met phosphorylation stimulated by HGF in A549 cells. (B) Anthraquinone derivatives failed to inhibit TPR-Met phosphorylation in BaF3/TPRMet cells. (C) Anthraquinone derivatives failed to inhibit c-Met phosphorylation in $\mathrm{MKN}-45$ cells.
MKN45 cells harbor amplified MET genes. The tested compounds, even at a concentration of $10 \mu \mathrm{M}$, failed to inhibit c-Met phosphorylation in the two cell lines (Figure 3B,C), indicating that the cellular inhibitory effects of these compounds are exclusively aimed at HGF-dependent activation. Given the distinctive advantage in cellular potency observed in this assay, compound $\mathbf{2 a}$ was selected as the lead for subsequent evaluation.

We then determined whether the inhibitory effect of $\mathbf{2 a}$ on HGF-mediated c-Met phosphorylation is associated with direct engagement with HGF. A surface plasmon resonance (SPR) assay was employed to assess the interaction between $\mathbf{2 a}$ and HGF, for which HGF was immobilized on GLH sensor chips. Encouragingly, compound 2a displayed high binding affinity with HGF, yielding an equilibrium dissociation constant $\left(K_{\mathrm{D}}\right)$ of $1.95 \mu \mathrm{M}$ (Figure 4). Compound 2a not only potentially inhibits intracellular c-Met kinase but also displays binding abilities with the extracellular HGF ligand. To further explore



Figure 4. Sensorgrams of compound 2a with immobilized HGF. Serial concentrations of compound $2 \mathrm{a}$ were injected over the HGFimmobilized GLH sensor chip surface. Real-time binding was recorded as response (RU) vs time. The SPR measurement yielded a $K_{\mathrm{D}}$ value of $1.95 \mu \mathrm{M}$ for compound $2 \mathrm{a}$. 
the mode of binding of compound 2a with HGF, a molecular docking simulation was performed. The theoretical result shows that compound $\mathbf{2 a}$ probably bound the $\mathrm{N}$-terminal heparin of HGF, forming stable electrostatic and cation $-\pi$ interactions with each other. The detailed information is provided as Supporting Information.

Many anthraquinone derivatives, including laxatives, antimalarials, and antineoplastics, have been developed as drugs, indicating that the scaffold has favorable pharmacological characteristics. ${ }^{18-20}$ Our study is the first to discover that anthraquinone derivatives are potent c-Met kinase inhibitors, which further enriches the structural space of small-molecule compounds. Among three cell lines tested, anthraquinone derivatives exclusively displayed potency toward A549 cells, in which the activation of c-Met kinase is stimulated by the HGF ligand, indicating that anthraquinone derivatives can block the extracellular HGF-dependent pathway. The most potent compound, 2a, displayed high binding affinity for HGF, with a $K_{\mathrm{D}}$ value of $1.95 \mu \mathrm{M}$. Until now, the marked expression of HGF has been detected in many lung cancers. HGF induces gefitinib resistance in the epidermal growth factor receptor of mutation-positive non-small cell lung cancer (NSCLC) by activating c-Met kinase signaling. ${ }^{9}$ Further studies of combination therapy with HGF antibodies and small-molecule kinase inhibitors are warranted in NSCLC patients with HGF-induced resistance. It is worth designing potential inhibitors targeting kinase and HGF in the meantime. Therefore, the dual roles of anthraquinone derivatives against c-Met kinase are clinically relevant and will shed new light on mechanism-based inhibitor design.

\section{EXPERIMENTAL PROCEDURES}

Kinase Enzyme-Linked Immunosorbent Assay (ELISA). Tyrosine kinase activities were evaluated according to the reported protocol. During the ELISA, $20 \mu \mathrm{g} / \mathrm{mL}$ poly(Glu, Tyr) 4:1 (Sigma) was precoated as a substrate on 96 -well plates. Approximately $50 \mu \mathrm{L}$ of a $5 \mu \mathrm{M}$ ATP solution diluted in kinase reaction buffer [50 mM HEPES (pH 7.4), $50 \mathrm{mM} \mathrm{MgCl}_{2}, 0.5 \mathrm{mM} \mathrm{MnCl}_{2}, 0.2 \mathrm{mM} \mathrm{Na}_{3} \mathrm{VO}_{4}$, and $1 \mathrm{mM}$ dithiothreitol] was then added to each well. Various concentrations of compounds diluted in $10 \mu \mathrm{L}$ of $1 \%$ dimethyl sulfoxide (DMSO) (v/v) were added to each reaction well; $1 \%$ DMSO (v/v) was used as the negative control.

The kinase reaction was initiated by the addition of purified tyrosine kinase proteins diluted in $40 \mu \mathrm{L}$ of a kinase reaction buffer solution. After incubation for $60 \mathrm{~min}$ at $37^{\circ} \mathrm{C}$, the plate was washed three times with phosphate-buffered saline (PBS) containing $0.1 \%$ Tween 20 (TPBS). Approximately $100 \mu \mathrm{L}$ of anti-phosphotyrosine (PY99) antibody [diluted $1: 500$ in $5 \mathrm{mg} / \mathrm{mL}$ bovine serum albumin (BSA) T-PBS] was added to the plate. After incubation for $30 \mathrm{~min}$ at $37^{\circ} \mathrm{C}$, the plate was washed three times. A solution of $100 \mu \mathrm{L}$ of horseradish peroxidase-conjugated goat anti-mouse immunoglobulin G (IgG) (diluted 1:2000 in $5 \mathrm{mg} / \mathrm{mL}$ BSA T-PBS) was added to the plate, which was then reincubated at $37{ }^{\circ} \mathrm{C}$ for $30 \mathrm{~min}$ and washed as described above. Finally, $100 \mu \mathrm{L}$ of a solution of $0.03 \% \mathrm{H}_{2} \mathrm{O}_{2}$ and 2 $\mathrm{mg} / \mathrm{mL} o$-phenylenediamine in $0.1 \mathrm{mM}$ citrate buffer $(\mathrm{pH} 5.5)$ was added to the plate. Samples were incubated at room temperature until color emerged. The reaction was terminated by the addition of $50 \mu \mathrm{L}$ of $2 \mathrm{M} \mathrm{H}_{2} \mathrm{SO}_{4}$, and the plate was read using a multiwell spectrophotometer (VERSAmax, Molecular Devices, Sunnyvale, CA) at $490 \mathrm{~nm}$. The inhibition rate (\%) was calculated using the expression $\left(1-A_{490} / A_{490}\right.$ control $) \times 100 \% . \mathrm{IC}_{50}$ values were calculated from the inhibition curves.

Western Blot Analysis. Cells were cultured under regular growth conditions to the exponential growth phase. A549 cells were serumstarved for $24 \mathrm{~h}$, treated with indicated anthraquinone derivatives for 2 $\mathrm{h}$ at $37^{\circ} \mathrm{C}$, incubated with HGF for $15 \mathrm{~min}$, and then lysed in $1 \times$ SDS (sodium dodecyl sulfate) sample buffer. MKN-45 and BaF3/TPR-Met cells were treated with indicated anthraquinone derivatives for $2 \mathrm{~h}$ at $37^{\circ} \mathrm{C}$ and then lysed in $1 \times$ SDS sample buffer. These cell lysates were subsequently resolved by $10 \%$ SDS-polyacrylamide gel electrophoresis and transferred to nitrocellulose membranes. The membranes were first probed with phospho-c-Met, c-Met (both from Cell Signaling Technology), and glyceraldehyde 3-phosphate dehydrogenase (KangChen Biotech) antibody and then with anti-rabbit or -mouse IgG horseradish peroxidase. Immunoreactive proteins were detected using ECL Plus (GE Healthcare), and images were captured with ImageQuant LAS 4010 (GE Healthcare).

Surface Plasmon Resonance (SPR) Analysis. The kinetics of the binding reactions between 2a and HGF was determined using an XPR36 (Bio-Rad) SPR apparatus. HGF was immobilized on GLH sensor chips according to the amine coupling protocol described in the application handbook. To assess real-time binding capacity, $200 \mu \mathrm{L}$ of 2a was injected over the sensor chip surface immobilized with HGF. The chip was then washed with HBS-EP buffer for $5 \mathrm{~min}$. The sensor chip surface was regenerated using $100 \mu \mathrm{L}$ of $\mathrm{NaCl}(2 \mathrm{M})$. All binding experiments were performed at $25{ }^{\circ} \mathrm{C}$ with a constant flow rate of 50 $\mu \mathrm{L}$ of HBS-EP/min. To correct for nonspecific binding and changes in the bulk refractive index, a blank channel without HGF was employed as the control for each experiment. Sensorgrams for all binding interactions were recorded in real time and analyzed after they had been subtracted from the blank channel. Changes in mass due to the binding response were recorded as resonance units. Binding kinetics and affinities were computed by equilibrium analysis using Bio-Rad ProteOn Manager version 2.0.1.

\section{ASSOCIATED CONTENT}

\section{Supporting Information}

Mode of binding of compound 2a with HGF fragment NK2, general methods, and synthesis and spectral characterization data of the target compounds. This material is available free of charge via the Internet at http://pubs.acs.org.

\section{AUTHOR INFORMATION}

\section{Corresponding Author}

*Telephone: +86-21-50806600. Fax: +86-21-50807188. E-mail: cluo@mail.shcnc.ac.cn (C.L.), mygeng@mail.shcnc.ac.cn (M.G.), or hliu@mail.shcnc.ac.cn (H.L.).

\section{Author Contributions}

Z.L., J.A., and X.D. contributed equally to this study.

\section{Funding}

We gratefully acknowledge the financial support from the 863 program (2012AA020302), State Key Program of Basic Research of China Grant 2009CB918502, the National Science and Technology Major Project "Key New Drug Creation and Manufacturing Program" (2013ZX09507-004), and the National Natural Science Foundation of China (81025017, 30725046, 81102461, 81021062, 91029704, and 21210003).

\section{Notes}

The authors declare no competing financial interest.

\section{REFERENCES}

(1) Yap, T. A.; de Bono, J. S. Targeting the HGF/c-Met axis: State of play. Mol. Cancer Ther. 2010, 9 (5), 1077-9.

(2) Liu, X.; Newton, R. C.; Scherle, P. A. Developing c-MET pathway inhibitors for cancer therapy: Progress and challenges. Trends Mol. Med. 2010, 16 (1), 37-45.

(3) Liu, X.; Newton, R. C.; Scherle, P. A. Development of c-MET pathway inhibitors. Expert Opin. Invest. Drugs 2011, 20 (9), 1225-41.

(4) Gherardi, E.; Birchmeier, W.; Birchmeier, C.; Woude, G. V. Targeting MET in cancer: Rationale and progress. Nat. Rev. Cancer 2012, 12 (2), 89-103. 
(5) Liang, Z.; Ding, X.; Ai, J.; Kong, X.; Chen, L.; Luo, C.; Geng, M.; Liu, H.; Chen, K.; Jiang, H. Discovering potent inhibitors against cMet kinase: Molecular design, organic synthesis and bioassay. Org. Biomol. Chem. 2012, 10, 421-30.

(6) Zhang, D.; Ai, J.; Liang, Z.; Li, C.; Peng, X.; Ji, Y.; Jiang, H.; Geng, M.; Luo, C.; Liu, H. Discovery of novel 2-aminopyridine-3carboxamides as c-Met kinase inhibitors. Bioorg. Med. Chem. 2012, 20 (17), 5169-80.

(7) Liu, X.; Newton, R. C.; Scherle, P. A. Development of c-MET pathway inhibitors. Expert Opin. Invest. Drugs 2011, 20 (9), 1225-41.

(8) Underiner, T. L.; Herbertz, T.; Miknyoczki, S. J. Discovery of small molecule c-Met inhibitors: Evolution and profiles of clinical candidates. Anti-Cancer Agents Med. Chem. 2010, 10 (1), 7-27.

(9) Okamoto, W.; Okamoto, I.; Tanaka, K.; Hatashita, E.; Yamada, Y.; Kuwata, K.; Yamaguchi, H.; Arao, T.; Nishio, K.; Fukuoka, M.; Janne, P. A.; Nakagawa, K. TAK-701, a humanized monoclonal antibody to hepatocyte growth factor, reverses gefitinib resistance induced by tumor-derived HGF in non-small cell lung cancer with an EGFR mutation. Mol. Cancer Ther. 2010, 9 (10), 2785-92.

(10) Underiner, T. L.; Herbertz, T.; Miknyoczki, S. J. Discovery of small molecule c-Met inhibitors: Evolution and profiles of clinical candidates. Anti-Cancer Agents Med. Chem. 2010, 10 (1), 7-27.

(11) Cui, J. J.; Tran-Dube, M.; Shen, H.; Nambu, M.; Kung, P. P.; Pairish, M.; Jia, L.; Meng, J.; Funk, L.; Botrous, I.; McTigue, M.; Grodsky, N.; Ryan, K.; Padrique, E.; Alton, G.; Timofeevski, S.; Yamazaki, S.; Li, Q.; Zou, H.; Christensen, J.; Mroczkowski, B.; Bender, S.; Kania, R. S.; Edwards, M. P. Structure based drug design of crizotinib (PF-02341066), a potent and selective dual inhibitor of mesenchymal-epithelial transition factor (c-MET) kinase and anaplastic lymphoma kinase (ALK). J. Med. Chem. 2011, 54 (18), 634263.

(12) Xie, Q. Q.; Xie, H. Z.; Ren, J. X.; Li, L. L.; Yang, S. Y. Pharmacophore modeling studies of type I and type II kinase inhibitors of Tie2. J. Mol. Graphics Modell. 2009, 27 (6), 751-8.

(13) Li, H. F.; Lu, T.; Zhu, T.; Jiang, Y. J.; Rao, S. S.; Hu, L. Y.; Xin, B. T.; Chen, Y. D. Virtual screening for Raf-1 kinase inhibitors based on pharmacophore model of substituted ureas. Eur. J. Med. Chem. 2009, 44 (3), 1240-9.

(14) Zou, J.; Xie, H. Z.; Yang, S. Y.; Chen, J. J.; Ren, J. X.; Wei, Y. Q. Towards more accurate pharmacophore modeling: Multicomplexbased comprehensive pharmacophore map and most-frequent-feature pharmacophore model of CDK2. J. Mol. Graphics Modell. 2008, 27 (4), $430-8$.

(15) Xiang, M.; Cao, Y.; Fan, W.; Chen, L.; Mo, Y. Computer-aided drug design: Lead discovery and optimization. Comb. Chem. High Throughput Screening 2012, 15 (4), 328-37.

(16) Liang, Z.; Zhang, D.; Ai, J.; Chen, L.; Wang, H.; Kong, X.; Zheng, M.; Liu, H.; Luo, C.; Geng, M.; Jiang, H.; Chen, K. Identification and synthesis of $\mathrm{N}^{\prime}$-(2-oxoindolin-3-ylidene)hydrazide derivatives against c-Met kinase. Bioorg. Med. Chem. Lett. 2011, 21 (12), 3749-54.

(17) Rickert, K. W.; Patel, S. B.; Allison, T. J.; Byrne, N. J.; Darke, P. L.; Ford, R. E.; Guerin, D. J.; Hall, D. L.; Kornienko, M.; Lu, J.; Munshi, S. K.; Reid, J. C.; Shipman, J. M.; Stanton, E. F.; Wilson, K. J.; Young, J. R.; Soisson, S. M.; Lumb, K. J. Structural Basis for Selective Small Molecule Kinase Inhibition of Activated c-Met. J. Biol. Chem. 2011, 286 (13), 11218-25.

(18) Zagotto, G.; Sissi, C.; Lucatello, L.; Pivetta, C.; Cadamuro, S. A.; Fox, K. R.; Neidle, S.; Palumbo, M. Aminoacyl-anthraquinone conjugates as telomerase inhibitors: Synthesis, biophysical and biological evaluation. J. Med. Chem. 2008, 51 (18), 5566-74.

(19) Morier-Teissier, E.; Boitte, N.; Helbecque, N.; Bernier, J. L.; Pommery, N.; Duvalet, J. L.; Fournier, C.; Hecquet, B.; Catteau, J. P.; Henichart, J. P. Synthesis and antitumor properties of an anthraquinone bisubstituted by the copper chelating peptide GlyGly-L-His. J. Med. Chem. 1993, 36 (15), 2084-90.

(20) Zembower, D. E.; Kam, C. M.; Powers, J. C.; Zalkow, L. H. Novel anthraquinone inhibitors of human leukocyte elastase and cathepsin G. J. Med. Chem. 1992, 35 (9), 1597-605. 